\title{
Universality of the discrete spectrum asymptotics of the three-particle Schrödinger operator on a lattice
}

\author{
Mukhiddin I. Muminov ${ }^{1}$, Tulkin H. Rasulov ${ }^{2}$ \\ ${ }^{1}$ Faculty of Scince, Universiti Teknologi Malaysia (UTM) \\ 81310 Skudai, Johor Bahru, Malaysia \\ ${ }^{2}$ Faculty of Physics and Mathematics, Bukhara State University \\ M. Ikbol str. 11, 200100 Bukhara, Uzbekistan \\ mmuminov@mail.ru,rth@mail.ru
}

PACS 02.30.Tb

DOI 10.17586/2220-8054-2015-6-2-280-293

In the present paper, we consider the Hamiltonian $H(K), K \in \mathbb{T}^{3}:=(-\pi ; \pi]^{3}$ of a system of three arbitrary quantum mechanical particles moving on the three-dimensional lattice and interacting via zero range potentials. We find a finite set $\Lambda \subset \mathbb{T}^{3}$ such that for all values of the total quasi-momentum $K \in \Lambda$ the operator $H(K)$ has infinitely many negative eigenvalues accumulating at zero. It is found that for every $K \in \Lambda$, the number $N(K ; z)$ of eigenvalues of $H(K)$ lying on the left of $z, z<0$, satisfies the asymptotic relation $\lim _{z \rightarrow-0} N(K ; z)|\log | z||^{-1}=\mathcal{U}_{0}$ with $0<\mathcal{U}_{0}<\infty$, independently on the cardinality of $\Lambda$.

Keywords: Three-particle Schrödinger operator, zero-range pair attractive potentials, Birman-Schwinger principle, the Efimov effect, discrete spectrum asymptotics.

Received: 18 January 2015

\section{Introduction}

We are going to discuss the following remarkable phenomenon of the spectral theory of the three-particle Schrödinger operators, known as the Efimov effect: if a system of three particles interacting through pair short-range potentials is such that none of the three twoparticle subsystems has bound states with negative energy, but at least two of them have a zero energy resonance, then this three-particle system has an infinite number of threeparticle bound states with negative energy accumulating at zero.

The Efimov effect was discussed in [1] for the first time. Since then, this problem has been studied on a physical level of rigor in [2,3]. A rigorous mathematical proof for the existence of Efimov's effect was originally carried out in [4], and subsequently, many works have been devoted to this subject, see for example [5-9]. The main result obtained by Sobolev [7] (see also [9]) is an asymptotics of the form $\mathcal{U}_{0}|\log | z||$ for the number $N(z)$ of eigenvalues on the left of $z, z<0$, where the coefficient $\mathcal{U}_{0}$ does not depend on the two-particle potentials $v_{\alpha}$ and is a positive function of the ratios $m_{1} / m_{2}$ and $m_{2} / m_{3}$ of the masses of the three particles.

In models of solid state physics [10-12] and also in lattice quantum field theory [13], one considers discrete Schrödinger operators, which are lattice analogs of the three-particle Schrödinger operator in continuous space. The presence of Efimov's effect for these operators was demonstrated at the physical level of rigor without a mathematical proof for a system of three identical quantum particles in $[10,11]$.

In the continuous case [14] (see also $[12,15]$ ), the energy of the center-of-mass motion can be separated out from the total Hamiltonian, that is, the energy operator can be split into the sum of the center-of-mass motion and the relative kinetic energy so that the 
three-particle bound states are eigenvectors of the relative kinetic energy operator. Therefore, Efimov's effect either exists or does not exist for all values of the total momentum simultaneously.

In lattice terms, the center-of-mass corresponds to a realization of the Hamiltonians as fibered operators, that is, as the direct integral of a family of operators $H(K)$ depending on the values of the total quasi-momentum $K \in \mathbb{T}^{3}:=(-\pi ; \pi]^{3}$ (see [12]). In this case, a bound state is an eigenvector of the operator $H(K)$ for some $K \in \mathbb{T}^{3}$. Typically, this eigenvector depends continuously on $K$. Therefore, Efimov's effect may exist only for some values of $K \in \mathbb{T}^{3}$.

The presence of the Efimov effect for three-particle discrete Schrödinger operators was proved in [16-18] and asymptotic formulas for the number of eigenvalues were obtained in $[16,17]$, which are analogous to the results of $[7,9]$.

In the present paper, we consider a system of three arbitrary quantum particles on the three-dimensional lattice interacting via zero-range potentials with the dispersion function of the form $\varepsilon(p)=\sum_{i=1}^{3}\left(1-\cos \left(n p^{(i)}\right)\right)$ with $n>1$. We denote by $\Lambda$ the set of points of $\mathbb{T}^{3}$ where the function $\varepsilon(\cdot)$ takes its (global) minimum. If at least two of the two-particle operators have a zero energy resonance and third one is non-negative, then we prove that for all $K \in \Lambda$, the three-particle discrete Schrödinger operator $H(K)$ has infinitely many negative eigenvalues accumulating at zero. Moreover, for any $K \in \Lambda$, we establish the asymptotic formula

$$
\lim _{z \rightarrow-0} N(K ; z)|\log | z||^{-1}=\mathcal{U}_{0} \quad\left(0<\mathcal{U}_{0}<\infty\right)
$$

where $N(K ; z)$ is the number of eigenvalues of $H(K)$ lying on the left of $z, z<0$.

It is surprising that the asymptotics for $N(K ; z)$ is the same for all $K \in \Lambda$ and is stable with respect to the number $n$. Recall that in all papers devoted to Efimov's effect for lattice systems, the existence of this effect has been proved only for the zero value of the quasi-momentum $(K=0)$ and for the case $n=1$. In [19], for all non-trivial values of total quasi-momentum $(K \neq 0)$, the finiteness of the discrete spectrum of a system of three bosons on a lattice was proven when the corresponding two-particle operator has a zero energy resonance.

The plan of this paper is as follows: Section 1 is an introduction to the whole work. In Section 2, the Hamiltonians of two- and three-particle systems are described as bounded self-adjoint operators in the corresponding Hilbert spaces and the main result of the paper is formulated. In Section 3, we discuss some results concerning threshold analysis of the two-particle operator $h_{\alpha}(k)$. In Section 4, we give a modification of the Birman-Schwinger principle for $H(K), K \in \mathbb{T}^{3}$. In Section 5 , we obtain an asymptotic formula for the number of negative eigenvalues of $H(K), K \in \Lambda$.

Throughout the present paper, we adopt the following conventions: For each $\delta>0$, the notation $U_{\delta}\left(p_{0}\right):=\left\{p \in \mathbb{T}^{3}:\left|p-p_{0}\right|<\delta\right\}$ stands for a $\delta$-neighborhood of the point $p_{0} \in \mathbb{T}^{3}$. The subscripts $\alpha, \beta, \gamma$ are pair-wisely different and takes values from $\{1,2,3\}$.

\section{Description of the three-particle operator}

Let $\mathbb{Z}^{3}$ be the three-dimensional lattice and $l_{2}\left(\left(\mathbb{Z}^{3}\right)^{m}\right)$ be the Hilbert space of squaresummable functions on $\left(\mathbb{Z}^{3}\right)^{m}, m=2,3$. The free Hamiltonian $\widehat{H}_{0}$ of a system of three arbitrary quantum mechanical particles on $\mathbb{Z}^{3}$ in the coordinate representation is usually 
associated with the following bounded self-adjoint operator on the Hilbert space $l_{2}\left(\left(\mathbb{Z}^{3}\right)^{3}\right)$ : $\left(\widehat{H}_{0} \widehat{\psi}\right)\left(x_{1}, x_{2}, x_{3}\right)=\sum_{s \in \mathbb{Z}^{3}}\left[\widehat{\varepsilon}_{1}(s) \widehat{\psi}\left(x_{1}+s, x_{2}, x_{3}\right)+\widehat{\varepsilon}_{2}(s) \widehat{\psi}\left(x_{1}, x_{2}+s, x_{3}\right)+\widehat{\varepsilon}_{3}(s) \widehat{\psi}\left(x_{1}, x_{2}, x_{3}+s\right)\right]$,

where $\widehat{\varepsilon}_{\alpha}(\cdot), \alpha=1,2,3$ are dispersion functions describing the particle transition from a site to a neighboring site defined by:

$$
\widehat{\varepsilon}_{\alpha}(s):=\left\{\begin{aligned}
\frac{3}{m_{\alpha}} & \text { as } \quad s=0 \\
-\frac{1}{2 m_{\alpha}} & \text { as } \quad s= \pm n e_{i}, i=1,2,3 \\
0 & \text { otherwise. }
\end{aligned}\right.
$$

Here, $m_{\alpha}>0$ are different numbers, having the meaning of a mass of the particle $\alpha$, $\alpha=1,2,3$, the elements $e_{i}, i=1,2,3$ are unit orts on $\mathbb{Z}^{3}$ and $n$ is a fixed positive integer with $n>1$.

It is easily seen that the function $\widehat{\varepsilon}_{\alpha}(\cdot)$ is even on $\mathbb{Z}^{3}$.

The three-particle Hamiltonian $\widehat{H}$ of the quantum-mechanical three particle systems with two-particle interactions $\widehat{v}_{\beta \gamma}, \beta, \gamma=1,2,3$ in the coordinate representation is a bounded perturbation of the free Hamiltonian $\widehat{H}_{0}$ :

$$
\widehat{H}=\widehat{H}_{0}-\widehat{V}_{1}-\widehat{V}_{2}-\widehat{V}_{3}
$$

where $\widehat{V}_{\alpha}, \alpha=1,2,3$ are multiplication operators on the Hilbert space $l_{2}\left(\left(\mathbb{Z}^{3}\right)^{3}\right)$

$$
\left(\widehat{V_{\alpha}} \widehat{\psi}\right)\left(x_{1}, x_{2}, x_{3}\right)=\widehat{v}_{\beta \gamma}\left(x_{\beta}-x_{\gamma}\right) \widehat{\psi}\left(x_{1}, x_{2}, x_{3}\right)=\mu_{\alpha} \delta_{x_{\beta} x_{\gamma}} \widehat{\psi}\left(x_{1}, x_{2}, x_{3}\right), \quad \widehat{\psi} \in l_{2}\left(\left(\mathbb{Z}^{3}\right)^{3}\right) .
$$

Here, $\mu_{\alpha}>0$ is the interaction energy of the particles $\beta$ and $\gamma, \delta_{x_{\beta} x_{\gamma}}$ is the Kronecker delta.

It is clear that the three-particle Hamiltonian $\widehat{H}$ is a bounded self-adjoint operator on the Hilbert space $l_{2}\left(\left(\mathbb{Z}^{3}\right)^{3}\right)$.

Similarly, as we introduced $\widehat{H}$, we introduce the corresponding two-particle Hamiltonians $\widehat{h}_{\alpha}, \alpha=1,2,3$ as bounded self-adjoint operators on the Hilbert space $l_{2}\left(\left(\mathbb{Z}^{3}\right)^{2}\right)$ :

$$
\widehat{h}_{\alpha}=\widehat{h}_{\alpha}^{0}-\widehat{v}_{\alpha}
$$

where

$$
\begin{gathered}
\left(\widehat{h}_{\alpha}^{0} \widehat{\varphi}\right)\left(x_{\beta}, x_{\gamma}\right)=\sum_{s \in \mathbb{Z}^{3}}\left[\widehat{\varepsilon}_{\beta}(s) \widehat{\varphi}\left(x_{\beta}+s, x_{\gamma}\right)+\widehat{\varepsilon}_{\gamma}(s) \widehat{\varphi}\left(x_{\beta}, x_{\gamma}+s\right)\right], \\
\left(\widehat{v}_{\alpha} \widehat{\varphi}\right)\left(x_{\beta}, x_{\gamma}\right)=\mu_{\alpha} \delta_{x_{\beta} x_{\gamma}} \widehat{\varphi}\left(x_{\beta}, x_{\gamma}\right), \quad \widehat{\varphi} \in l_{2}\left(\left(\mathbb{Z}^{3}\right)^{2}\right) .
\end{gathered}
$$

Let us rewrite our operators in the momentum representation. We denote by $\mathbb{T}^{3}$ the three-dimensional torus, the cube $(-\pi, \pi]^{3}$ with appropriately identified sides and $L_{2}\left(\left(\mathbb{T}^{3}\right)^{m}\right)$ be the Hilbert space of square integrable (complex) functions defined on $\left(\mathbb{T}^{3}\right)^{m}, m=1,2,3$.

Let $\mathcal{F}_{m}: L_{2}\left(\left(\mathbb{T}^{3}\right)^{m}\right) \rightarrow l_{2}\left(\left(\mathbb{Z}^{3}\right)^{m}\right), m=2,3$ be the discrete Fourier transform. The three-particle Hamiltonian in the momentum representation is given by the bounded selfadjoint operator on the Hilbert space $L_{2}\left(\left(\mathbb{T}^{3}\right)^{3}\right)$ as follows $\widetilde{H}=\mathcal{F}_{3}^{-1} \widehat{H} \mathcal{F}_{3}$. Introducing the total quasi-momentum $K \in \mathbb{T}^{3}$ the operator $\widetilde{H}$ can be decomposed into von Neumann direct integrals of the family of bounded self-adjoint operators $\widetilde{H}(K), K \in \mathbb{T}^{3}$. The operator $\widetilde{H}(K), K \in \mathbb{T}^{3}$ is called the three-particle discrete Schrödinger operator, which is unitarily 
equivalent (see [16-18]) to the family of bounded self-adjoint operators $H(K), K \in \mathbb{T}^{3}$, acting on the Hilbert space $L_{2}\left(\left(\mathbb{T}^{3}\right)^{2}\right)$ according to the formula:

$$
H(K)=H_{0}(K)-V_{1}-V_{2}-V_{3},
$$

where $H_{0}(K)$ is the multiplication operator by the function:

$$
\mathcal{E}_{K}(p, q):=\varepsilon_{1}(p)+\varepsilon_{2}(q)+\varepsilon_{3}(K-p-q),
$$

where

$$
\varepsilon_{\alpha}(p):=\frac{1}{m_{\alpha}} \varepsilon(p), \quad \varepsilon(p):=\sum_{i=1}^{3}\left(1-\cos \left(n p^{(i)}\right)\right)
$$

and

$$
\begin{gathered}
\left(V_{1} f\right)(p, q)=\frac{\mu_{1}}{(2 \pi)^{3}} \int_{\mathbb{T}^{3}} f(p, s) d s, \quad\left(V_{2} f\right)(p, q)=\frac{\mu_{2}}{(2 \pi)^{3}} \int_{\mathbb{T}^{3}} f(s, q) d s, \\
\left(V_{3} f\right)(p, q)=\frac{\mu_{3}}{(2 \pi)^{3}} \int_{\mathbb{T}^{3}} f(s, p+q-s) d s .
\end{gathered}
$$

Similarly, the study of the spectral properties of the $\widetilde{h}_{\alpha}=\mathcal{F}_{2}^{-1} \widehat{h}_{\alpha} \mathcal{F}_{2}$ can be reduced to the study of the spectral properties of the family of bounded self-adjoint operators $h_{\alpha}(k)$, $k \in \mathbb{T}^{3}$, corresponding to the two-particle lattice Hamiltonians on the Hilbert space $L_{2}\left(\mathbb{T}^{3}\right)$ :

$$
h_{\alpha}(k)=h_{\alpha}^{0}(k)-v_{\alpha} .
$$

The non-perturbed operator $h_{\alpha}^{0}(k)$ is the multiplication operator on $L_{2}\left(\mathbb{T}^{3}\right)$ by the function:

$$
E_{k}^{(\alpha)}(p):=\varepsilon_{\beta}(p)+\varepsilon_{\gamma}(k-p), \quad \beta<\gamma, \quad \alpha, \beta, \gamma=1,2,3 .
$$

The perturbation $v_{\alpha}$ is an integral operator of rank one on $L_{2}\left(\mathbb{T}^{3}\right)$ :

$$
\left(v_{\alpha} f\right)(p)=\frac{\mu_{\alpha}}{(2 \pi)^{3}} \int_{\mathbb{T}^{3}} f(s) d s .
$$

Therefore, by the Weyl theorem, the continuous spectrum $\sigma_{\text {cont }}\left(h_{\alpha}(k)\right)$ of the operator $h_{\alpha}(k)$ coincides with the spectrum of $\sigma\left(h_{\alpha}^{0}(k)\right)$ of $h_{\alpha}^{0}(k)$. More specifically:

$$
\sigma_{\text {cont }}\left(h_{\alpha}(k)\right)=\left[E_{\min }^{(\alpha)}(k) ; E_{\max }^{(\alpha)}(k)\right],
$$

where

$$
E_{\min }^{(\alpha)}(k):=\min _{p \in \mathbb{T}^{3}} E_{k}^{(\alpha)}(p) \quad \text { and } \quad E_{\max }^{(\alpha)}(k):=\max _{p \in \mathbb{T}^{3}} E_{k}^{(\alpha)}(p)
$$

\section{Formulation of the main results}

We denote by $\sigma_{\text {ess }}(\cdot)$ and $\sigma_{\text {disc }}(\cdot)$ the essential spectrum and the discrete spectrum of a bounded self-adjoint operator, respectively.

The following theorem, $[17,18]$, describes the location of the essential spectrum of the operator $H(K)$.

Theorem 3.1. For the essential spectrum of $H(K)$, the following equality holds:

$$
\sigma_{\text {ess }}(H(K))=\bigcup_{\alpha=1}^{3} \bigcup_{p \in \mathbb{T}^{3}}\left\{\sigma_{\text {disc }}\left(h_{\alpha}(K-p)\right)+\varepsilon_{\alpha}(p)\right\} \cup\left[\mathcal{E}_{\min }(K) ; \mathcal{E}_{\max }(K)\right],
$$


where

$$
\mathcal{E}_{\min }(K):=\min _{p, q \in \mathbb{T}^{3}} \mathcal{E}_{K}(p, q) \quad \text { and } \quad \mathcal{E}_{\max }(K):=\max _{p, q \in \mathbb{T}^{3}} \mathcal{E}_{K}(p, q)
$$

Let us consider the following subset of $\mathbb{T}^{3}$ :

$$
\Lambda:=\left\{\left(p^{(1)}, p^{(2)}, p^{(3)}\right): p^{(i)} \in\left\{0, \pm \frac{2}{n} \pi ; \pm \frac{4}{n} \pi ; \ldots ; \pm \frac{n^{\prime}}{n} \pi\right\} \cup \Pi_{n}, i=1,2,3\right\}
$$

where

$$
n^{\prime}:=\left\{\begin{array}{ll}
n-2, & \text { if } n \text { is even; } \\
n-1, & \text { if } n \text { is odd, }
\end{array} \text { and } \quad \Pi_{n}:= \begin{cases}\{\pi\}, & \text { if } n \text { is even; } \\
\emptyset, & \text { if } n \text { is odd }\end{cases}\right.
$$

Direct calculation shows that the cardinality of $\Lambda$ is equal to $n^{3}$. It is easy to verify that for any $K \in \Lambda$, the function $\mathcal{E}_{K}(\cdot, \cdot)$ has non-degenerate zero minima at the points of $\Lambda \times \Lambda$, that is, $\mathcal{E}_{\min }(K)=0$ for $K \in \Lambda$.

Since $\mathbf{0}=(0,0,0) \in \Lambda$, the definition of the functions $E_{k}^{(\alpha)}(\cdot)$ and $\mathcal{E}_{K}(\cdot, \cdot)$ imply the identities $h_{\alpha}(\mathbf{0}) \equiv h_{\alpha}(k)$ and $H(\mathbf{0}) \equiv H(K)$ for all $k, K \in \Lambda$.

Let $C\left(\mathbb{T}^{3}\right)$ and $L_{1}\left(\mathbb{T}^{3}\right)$ be the Banach spaces of continuous and integrable functions on $\mathbb{T}^{3}$, respectively. Let $G_{\alpha}$ be the integral operator on $C\left(\mathbb{T}^{3}\right)$ with the kernel:

$$
G_{\alpha}(p, s)=\frac{\mu_{\alpha}}{(2 \pi)^{3}} \frac{m_{\beta} m_{\gamma}}{m_{\beta}+m_{\gamma}} \frac{1}{\varepsilon(s)} .
$$

Definition 3.2. The operator $h_{\alpha}(\mathbf{0})$ is said to have a zero energy resonance if the number 1 is an eigenvalue of the operator $G_{\alpha}$. If the number 1 isn't an eigenvalue of the operator $G_{\alpha}$, then we say that $z=0$ is a regular-type point for the operator $h_{\alpha}(\mathbf{0})$.

We note that in Definition 3.2 the requirement of the existence of the eigenfunction $\varphi_{\alpha} \in C\left(\mathbb{T}^{3}\right)$ corresponding to the eigenvalue 1 of $G_{\alpha}$ corresponds to the existence of a solution of $h_{\alpha}(\mathbf{0}) f_{\alpha}=0$, and this solution does not belong to $L_{2}\left(\mathbb{T}^{3}\right)$. More precisely, if the operator $h_{\alpha}(\mathbf{0})$ has a zero energy resonance, then the function:

$$
f_{\alpha}(p)=\varphi_{\alpha}(p)(\varepsilon(p))^{-1},
$$

is a solution (up to a constant factor) of the Schrödinger equation $h_{\alpha}(\mathbf{0}) f_{\alpha}=0$ and $f_{\alpha} \in$ $L_{1}\left(\mathbb{T}^{3}\right) \backslash L_{2}\left(\mathbb{T}^{3}\right)$ (see Lemma 4.4).

We set:

$$
\mu_{\alpha}^{0}:=8 \pi^{3} \frac{m_{\beta}+m_{\gamma}}{m_{\beta} m_{\gamma}}\left(\int_{\mathbb{T}^{3}} \frac{d s}{\varepsilon(s)}\right)^{-1}, \quad \alpha=1,2,3 .
$$

Simple calculation shows that the operator $h_{\alpha}(\mathbf{0})$ has a zero energy resonance if and only if $\mu_{\alpha}=\mu_{\alpha}^{0}$ (see Lemma 4.2).

For $K \in \mathbb{T}^{3}$, let us denote by $\tau_{\text {ess }}(K)$ the bottom of the essential spectrum of $H(K)$ and by $N(K ; z)$ the number of eigenvalues of $H(K)$ lying on the left of $z, z<\tau_{\text {ess }}(K)$. It is clear that $N(\mathbf{0} ; z)=N(K ; z)$ for any $K \in \Lambda$.

Since the operator $h_{\alpha}(\mathbf{0})$ has no negative eigenvalues for all $\mu_{\alpha} \leq \mu_{\alpha}^{0}$ (see Lemma 4.3), the operator $h_{\alpha}(\mathbf{0})$ is non-negative for all $\mu_{\alpha} \leq \mu_{\alpha}^{0}$. Then, by Theorem 1 of [21], the operator $h_{\alpha}(k)$ is non-negative for all $\mu_{\alpha} \leq \mu_{\alpha}^{0}$ and $k \in \mathbb{T}^{3}$. Hence, the assertion $\mathcal{E}_{\min }(K)=0$, $K \in \Lambda$ implies $\tau_{\text {ess }}(K)=0$ for $K \in \Lambda$ and $\mu_{\alpha} \leq \mu_{\alpha}^{0}$.

The main result of the present paper is given in the following theorem. 
Theorem 3.3. Assume $\mu_{\alpha}=\mu_{\alpha}^{0}, \mu_{\beta}=\mu_{\beta}^{0}$ and $\mu_{\gamma} \leq \mu_{\gamma}^{0}$. Then the operator $H(\mathbf{0})$ has infinitely many negative eigenvalues accumulating at zero and the function $N(\mathbf{0} ; \cdot)$ obeys the relation:

$$
\lim _{z \rightarrow-0} \frac{N(\mathbf{0} ; z)}{|\log | z||}=\mathcal{U}_{0}, \quad 0<\mathcal{U}_{0}<\infty
$$

Remark 3.4. The constant $\mathcal{U}_{0}$ does not depend on the interaction energies $\mu_{\alpha}, \alpha=1,2,3$; it is positive and depends only on the ratios $m_{\beta} / m_{\alpha}, \alpha \neq \beta, \alpha, \beta=1,2,3$ between the masses.

Remark 3.5. Clearly, by equality (3.2), the infinite cardinality of the negative discrete spectrum of $H(\mathbf{0})$ follows automatically from the positivity of $\mathcal{U}_{0}$.

Remark 3.6. It is surprising that the asymptotics (3.2) don't depend on the cardinality of $\Lambda$, that is, these asymptotics are the same for all $n \in \mathbb{N}$. Since $\left.\Lambda\right|_{n=1}=\{\mathbf{0}\}$ in fact, Theorem 3.3 was proved in [17] for $n=1$.

\section{Threshold analysis of the two-particle operator $h_{\alpha}(k)$}

In this section, we study the spectral properties of the two-particle discrete Schrödinger operator $h_{\alpha}(k)$.

For any $\mu_{\alpha}>0, k \in \mathbb{T}^{3}$ and $z \in \mathbb{C} \backslash \sigma_{\text {cont }}\left(h_{\alpha}(k)\right)$ we define the function (the Fredholm determinant associated with the operator $\left.h_{\alpha}(k)\right)$ :

$$
\Delta_{\alpha}(k ; z):=1-\frac{\mu_{\alpha}}{(2 \pi)^{3}} \int_{\mathbb{T}^{3}} \frac{d s}{E_{k}^{(\alpha)}(s)-z} .
$$

Note that the function $\Delta_{\alpha}(\cdot ; \cdot)$ is analytic in $\mathbb{T}^{3} \times\left(\mathbb{C} \backslash \sigma_{\text {cont }}\left(h_{\alpha}(k)\right)\right)$.

The following lemma is a simple consequence of the Birman-Schwinger principle and the Fredholm theorem.

Lemma 4.1. The number $z \in \mathbb{C} \backslash \sigma_{\text {cont }}\left(h_{\alpha}(k)\right)$ is an eigenvalue of the operator $h_{\alpha}(k)$, $k \in \mathbb{T}^{3}$ if and only if $\Delta_{\alpha}(k ; z)=0$. for $k \in \Lambda$.

We remark that from the definition of $E_{k}^{(\alpha)}(\cdot)$, it follows that $\Delta_{\alpha}(\mathbf{0} ; 0)=\Delta_{\alpha}(k ; 0)$

Lemma 4.2. The following statements are equivalent:

(i) the operator $h_{\alpha}(\mathbf{0})$ has a zero energy resonance;

(ii) $\Delta_{\alpha}(\mathbf{0} ; 0)=0$;

(iii) $\mu_{\alpha}=\mu_{\alpha}^{0}$.

For the proof of Lemma 4.2, see Lemma 5.3 of [17].

Lemma 4.3. The operator $h_{\alpha}(0)$ has no negative eigenvalues for all $\mu_{\alpha} \leq \mu_{\alpha}^{0}$.

Proof. Since the function $\Delta_{\alpha}(\mathbf{0} ; \cdot)$ is decreasing on $(-\infty ; 0)$, we have

$$
\Delta_{\alpha}(\mathbf{0} ; z)>\Delta_{\alpha}(\mathbf{0} ; 0)
$$

for all $z<0$. Definition of $\mu_{\alpha}^{0}$ implies $\Delta_{\alpha}(\mathbf{0} ; 0) \geq 0$ for all $\mu_{\alpha} \leq \mu_{\alpha}^{0}$. Hence by inequality (4.1) we have $\Delta_{\alpha}(\mathbf{0} ; z)>0$ for any $\mu_{\alpha} \leq \mu_{\alpha}^{0}$ and $z<0$. By Lemma 4 .1, it means that the operator $h_{\alpha}(\mathbf{0})$ has no negative eigenvalues for all $\mu_{\alpha} \leq \mu_{\alpha}^{0}$. 
set:

In the sequel, we denote by $C_{1}, C_{2}, C_{3}$ different positive numbers and for $\delta>0$ we

$$
\mathbb{T}_{\delta}:=\mathbb{T}^{3} \backslash \bigcup_{p^{\prime} \in \Lambda} U_{\delta}\left(p^{\prime}\right)
$$

Lemma 4.4. If $h_{\alpha}(\mathbf{0})$ has a zero energy resonance, then the function $f_{\alpha}(p)=\varphi_{\alpha}(p)(\varepsilon(p))^{-1}$ obeys the equation $h_{\alpha}(\mathbf{0}) f_{\alpha}=0$ and $f_{\alpha} \in L_{1}\left(\mathbb{T}^{3}\right) \backslash L_{2}\left(\mathbb{T}^{3}\right)$, where the function $\varphi_{\alpha} \in C\left(\mathbb{T}^{3}\right)$ is a unique (up to a constant factor) solution of $G_{\alpha} \varphi_{\alpha}=\varphi_{\alpha}$ satisfying the condition $\varphi_{\alpha}(\mathbf{0}) \neq 0$.

Proof. Let the operator $h_{\alpha}(\mathbf{0})$ have a zero energy resonance. One can see that the function $f_{\alpha}$ defined in Lemma 4.4 satisfies $h_{\alpha}(\mathbf{0}) f_{\alpha}=0$. Let us show that $f_{\alpha} \in L_{1}\left(\mathbb{T}^{3}\right) \backslash L_{2}\left(\mathbb{T}^{3}\right)$. First we recall that the solution of $G_{\alpha} \varphi_{\alpha}=\varphi_{\alpha}$ is equal to $\varphi_{\alpha}(p) \equiv 1$ (up to constant factor). The definition of the function $\varepsilon(\cdot)$ implies the existence of positive constants $C_{1}, C_{2}, C_{3}$ and $\delta$ such that:

$$
\begin{gathered}
C_{1}\left|q-p^{\prime}\right|^{2} \leq \varepsilon(q) \leq C_{2}\left|q-p^{\prime}\right|^{2}, \quad q \in U_{\delta}\left(p^{\prime}\right), \quad p^{\prime} \in \Lambda \\
\varepsilon(q) \geq C_{3}, \quad q \in \mathbb{T}_{\delta} .
\end{gathered}
$$

Using the estimates (4.2) and (4.3) we have:

$$
\begin{aligned}
& \int_{\mathbb{T}^{3}}\left|f_{\alpha}(s)\right|^{2} d s \geq \int_{U_{\delta}(\mathbf{0})} \frac{d s}{\varepsilon^{2}(s)} \geq C_{2} \int_{U_{\delta}(\mathbf{0})} \frac{d s}{|s|^{4}}=\infty ; \\
& \int_{\mathbb{T}^{3}}\left|f_{\alpha}(s)\right| d s=\sum_{p^{\prime} \in \Lambda} \int_{U_{\delta}\left(p^{\prime}\right)} \frac{d s}{\varepsilon(s)}+\int_{\mathbb{T}_{\delta}} \frac{d s}{\varepsilon(s)} \leq C_{1} \sum_{p^{\prime} \in \Lambda_{U_{\delta}\left(p^{\prime}\right)}} \int_{\left|s-p^{\prime}\right|^{2}} \frac{d s}{\mid s}<\infty .
\end{aligned}
$$

Therefore, $f_{\alpha} \in L_{1}\left(\mathbb{T}^{3}\right) \backslash L_{2}\left(\mathbb{T}^{3}\right)$.

We denote:

$$
m_{\beta \gamma}:=\frac{m_{\beta}+m_{\gamma}}{m_{\beta} m_{\gamma}}, \quad n_{\alpha}:=\frac{m_{1}+m_{2}+m_{3}}{m_{\alpha}\left(m_{\beta}+m_{\gamma}\right)} .
$$

Now, we formulate a lemma (zero energy expansion for the Fredholm determinant, leading to behaviors of the zero energy resonance), which is important in the proof of Theorem 3.3, that is, the asymptotics (3.2).

Lemma 4.5. Let $\mu_{\alpha}=\mu_{\alpha}^{0}$ and $K, p^{\prime} \in \Lambda$. Then, the following decomposition:

$$
\Delta_{\alpha}\left(K-p ; z-\varepsilon_{\alpha}(p)\right)=\frac{n \mu_{\alpha}^{0} m_{\beta \gamma}^{3 / 2}}{2 \pi} \sqrt{n_{\alpha}\left|p-p^{\prime}\right|^{2}-\frac{2 z}{n^{2}}}+O\left(\left|p-p^{\prime}\right|^{2}\right)+O(|z|)
$$

holds for $\left|p-p^{\prime}\right| \rightarrow 0$ and $z \rightarrow-0$.

Proof. Let us sketch the main idea of the proof. Take a sufficiently small $\delta>0$ such that $U_{\delta}\left(p^{\prime}\right) \cap U_{\delta}\left(q^{\prime}\right)=\emptyset$ for all $q^{\prime} \in \Lambda$ with $q^{\prime} \neq p^{\prime}$. Let $\mu_{\alpha}=\mu_{\alpha}^{0}$ and $K, p^{\prime} \in \Lambda$. Using the additivity of the integral, we rewrite the function $\Delta_{\alpha}\left(K-p ; z-\varepsilon_{\alpha}(p)\right)$ as:

$$
\begin{aligned}
& \Delta_{\alpha}\left(K-p ; z-\varepsilon_{\alpha}(p)\right)= \\
& 1-\frac{\mu_{\alpha}^{0}}{(2 \pi)^{3}}\left(\sum_{q^{\prime} \in \Lambda} \int_{U_{\delta}\left(q^{\prime}\right)} \frac{d s}{E_{p}^{(\alpha)}(s)+\varepsilon_{\alpha}(p)-z}+\int_{\mathbb{T}_{\delta}} \frac{d s}{E_{p}^{(\alpha)}(s)+\varepsilon_{\alpha}(p)-z}\right) .
\end{aligned}
$$


Since the function $\mathcal{E}_{K}(\cdot, \cdot)$ has non-degenerate zero minima at the points $\left(p^{\prime}, q^{\prime}\right)$, $p^{\prime}, q^{\prime} \in \Lambda$, analysis similar to [17] shows that:

$$
\begin{aligned}
& \int_{U_{\delta}\left(q^{\prime}\right)} \frac{d s}{E_{p}^{(\alpha)}(-s)+\varepsilon_{\alpha}(p)-z}= \int_{U_{\delta}\left(q^{\prime}\right)} \frac{d s}{E_{p^{\prime}}^{(\alpha)}(-s)+\varepsilon_{\alpha}\left(p^{\prime}\right)}- \\
& \frac{4 \pi^{2} m_{\beta \gamma}^{3 / 2}}{n^{2}} \sqrt{n_{\alpha}\left|p-p^{\prime}\right|^{2}-\frac{2 z}{n^{2}}}+O\left(\left|p-p^{\prime}\right|^{2}\right)+O(|z|) ; \\
& \int_{\mathbb{T}_{\delta}} \frac{d s}{E_{p}^{(\alpha)}(s)+\varepsilon_{\alpha}(p)-z}=\int_{\mathbb{T}_{\delta}} \frac{d s}{E_{p^{\prime}}^{(\alpha)}(s)+\varepsilon_{\alpha}\left(p^{\prime}\right)}+O\left(\left|p-p^{\prime}\right|^{2}\right)+O(|z|)
\end{aligned}
$$

as $\left|p-p^{\prime}\right| \rightarrow 0$ and $z \rightarrow-0$. Substituting the last two expressions into (4.4), we obtain:

$\Delta_{\alpha}\left(K-p ; z-\varepsilon_{\alpha}(p)\right)=\Delta_{\alpha}\left(K-p^{\prime} ; 0\right)+\frac{n \mu_{\alpha}^{0} m_{\beta \gamma}^{3 / 2}}{2 \pi} \sqrt{n_{\alpha}\left|p-p^{\prime}\right|^{2}-\frac{2 z}{n^{2}}}+O\left(\left|p-p^{\prime}\right|^{2}\right)+O(|z|)$ as $\left|p-p^{\prime}\right| \rightarrow 0$ and $z \rightarrow-0$. Now, the equality $\mu_{\alpha}=\mu_{\alpha}^{0}$, that is, $\Delta_{\alpha}\left(K-p^{\prime} ; 0\right)=0$ completes the proof of Lemma 4.5 .

Corollary 4.6. Let $\mu_{\alpha}=\mu_{\alpha}^{0}$ and $K \in \Lambda$. For some $C_{1}, C_{2}, C_{3}>0$ and $\delta>0$ the following inequalities hold:

(i) $C_{1}\left|p-p^{\prime}\right| \leq \Delta_{\alpha}\left(K-p ;-\varepsilon_{\alpha}(p)\right) \leq C_{2}\left|p-p^{\prime}\right|, p \in U_{\delta}\left(p^{\prime}\right), p^{\prime} \in \Lambda$;

(ii) $\Delta_{\alpha}\left(K-p ;-\varepsilon_{\alpha}(p)\right) \geq C_{3}, p \in \mathbb{T}_{\delta}$.

Proof. Lemma 4.5 yields the assertion (i) for some positive numbers $C_{1}, C_{2}$. The positivity and continuity of the function $\Delta_{\alpha}\left(\cdot ;-\varepsilon_{\alpha}(\cdot)\right)$ on the compact set $\mathbb{T}_{\delta}$ imply the assertion (ii).

\section{The Birman-Schwinger principle}

For a bounded self-adjoint operator $A$ acting in the Hilbert space $\mathcal{R}$, we define the number $n(\gamma, A)$ as follows:

$$
n(\gamma, A)=\sup \{\operatorname{dim} F:(A u, u)>\gamma, u \in F \subset \mathcal{R},\|u\|=1\} .
$$

The number $n(\gamma, A)$ is equal to infinity if $\gamma<\max \sigma_{\text {ess }}(A)$; if $n(\gamma, A)$ is finite, then it is equal to the number of the eigenvalues of $A$ larger than $\gamma$.

By the definition of $N(K ; z)$, we have:

$$
N(K ; z)=n(-z,-H(K)),-z>-\tau_{\text {ess }}(K) .
$$

Let $\mu_{\alpha}>0$ and $K \in \mathbb{T}^{3}$. Then we have $\Delta_{\alpha}\left(K-p ; z-\varepsilon_{\alpha}(p)\right)>0$ for any $p \in \mathbb{T}^{3}$ and $z<\tau_{\text {ess }}(K)$.

In what follows, we deal with the operators in various spaces of vector-valued functions. They will be denoted by bold letters and will be written in matrix form.

Let $\Omega \subset \mathbb{R}^{3}$ be the measurable set and $L_{2}^{(m)}(\Omega)$ be the Hilbert space of $m$-component vector functions $w=\left(w_{1}, \ldots, w_{m}\right), w_{i} \in L_{2}(\Omega), i=1, \ldots, m$.

In our analysis of the discrete spectrum of $H(K)$, the crucial role is played by the $3 \times 3$ self-adjoint block operator matrix $\widehat{\mathbf{T}}(K ; z), z<\tau_{\text {ess }}(K)$ acting on $L_{2}^{(3)}\left(\mathbb{T}^{3}\right)$ with the 
entries $\widehat{T}_{\alpha \beta}(K ; z)$, where $\widehat{T}_{\alpha \beta}(K ; z), \alpha \leq \beta$ is the integral operator on $L_{2}\left(\mathbb{T}^{3}\right)$ with kernel $\widehat{T}_{\alpha \alpha}(K ; z ; \cdot, \cdot)$ :

$$
\begin{aligned}
& \widehat{T}_{\alpha \alpha}(K ; z ; p, q)=0 ; \\
& \widehat{T}_{12}(K ; z ; p, q)=\frac{\sqrt{\mu_{1} \mu_{2}}}{\sqrt{\Delta_{1}\left(K-p ; z-\varepsilon_{1}(p)\right)}} \frac{\left(\mathcal{E}_{K}(p, q)-z\right)^{-1}}{\sqrt{\Delta_{2}\left(K-q ; z-\varepsilon_{2}(q)\right)}}, \\
& \widehat{T}_{13}(K ; z ; p, q)=\frac{\sqrt{\mu_{1} \mu_{3}}}{\sqrt{\Delta_{1}\left(K-p ; z-\varepsilon_{1}(p)\right)}} \frac{\left(\mathcal{E}_{K}(p, q-p)-z\right)^{-1}}{\sqrt{\Delta_{3}\left(q ; z-\varepsilon_{3}(K-q)\right)}}, \\
& \widehat{T}_{23}(K ; z ; p, q)=\frac{\sqrt{\mu_{2} \mu_{3}}}{\sqrt{\Delta_{2}\left(K-p ; z-\varepsilon_{2}(p)\right)}} \frac{\left(\mathcal{E}_{K}(q-p, p)-z\right)^{-1}}{\sqrt{\Delta_{3}\left(q ; z-\varepsilon_{3}(K-q)\right)}},
\end{aligned}
$$

and for $\alpha>\beta$ the operator $\widehat{T}_{\alpha \beta}(K ; z)$ is the adjoint operator to $\widehat{T}_{\beta \alpha}(K ; z)$.

The following lemma is a realization of the well-known Birman-Schwinger principle for three-particle Schrödinger operators on a lattice (see $[7,16,17])$.

Lemma 5.1. For $z<\tau_{\mathrm{ess}}(K)$ the operator $\widehat{\mathbf{T}}(K ; z)$ is compact and continuous in $z$ and

$$
N(K ; z)=n(1, \widehat{\mathbf{T}}(K ; z)) .
$$

For the proof of the lemma, we refer to [17].

\section{Asymptotics for the number of negative eigenvalues of $H(0)$}

In this section, we derive the asymptotic relation (3.2) for the number of negative eigenvalues of $H(\mathbf{0})$.

First, we recall that $\widehat{\mathbf{T}}(\mathbf{0} ; z) \equiv \widehat{\mathbf{T}}(K ; z)$ for all $K \in \Lambda$. Let $\mathbb{S}^{2}$ be the unit sphere in $\mathbb{R}^{3}$ and $\sigma=L_{2}\left(\mathbb{S}^{2}\right)$. As we shall see, the discrete spectrum asymptotics of the operator $\widehat{\mathbf{T}}(\mathbf{0} ; z)$ as $z \rightarrow-0$ is determined by the integral operator $\mathbf{S}_{\mathbf{r}}, \mathbf{r}=1 / 2|\log | z||$ in $L_{2}\left((0, \mathbf{r}), \sigma^{(3)}\right)$ with the kernel $S_{\alpha \beta}(y, t), y=x-x^{\prime}, x, x^{\prime} \in(0, \mathbf{r}), t=\langle\xi, \eta\rangle, \xi, \eta \in \mathbb{S}^{2}$, where:

$$
\begin{aligned}
& S_{\alpha \alpha}(y, t)=0 ; \quad S_{\alpha \beta}(y, t)=\frac{1}{4 \pi^{2}} \frac{u_{\alpha \beta}}{\cosh \left(y+r_{\alpha \beta}\right)+s_{\alpha \beta} t} ; \\
& u_{\alpha \beta}=k_{\alpha \beta}\left(\frac{m_{\beta \gamma}^{-1} m_{\alpha \gamma}^{-1}}{n_{\alpha} n_{\beta}}\right)^{1 / 4}, \quad r_{\alpha \beta}=\frac{1}{2} \log \frac{m_{\beta \gamma}}{m_{\alpha \gamma}}, \quad s_{\alpha \beta}=\frac{\left(m_{\alpha \gamma} m_{\beta \gamma}\right)^{1 / 2}}{m_{\gamma}},
\end{aligned}
$$

$k_{\alpha \beta}$ being such that $k_{\alpha \beta}=1$ if both subsystems $\alpha$ and $\beta$ have zero energy resonances, otherwise, $k_{\alpha \beta}=0$. The eigenvalue asymptotics for the operator $\mathbf{S}_{\mathbf{r}}$ have been studied in detail by Sobolev [7], by employing an argument used in the calculation of the canonical distribution of Toeplitz operators.

Let us recall some results of [7] which are important in our work.

The coefficient in asymptotics $(3.2)$ of $N(\mathbf{0} ; z)$ will be expressed by means of the self-adjoint integral operator $\widehat{\mathbf{S}}(\theta), \theta \in \mathbb{R}$, in the space $\sigma^{(3)}$, whose kernel is of the form:

$$
\widehat{S}_{\alpha \alpha}(\theta, t)=0 ; \quad \widehat{S}_{\alpha \beta}(\theta, t)=\frac{1}{4 \pi^{2}} u_{\alpha \beta} e^{i r_{\alpha \beta} \theta} \frac{\sinh \left[\theta \arccos s_{\alpha \beta} t\right]}{\sqrt{1-s_{\alpha \beta}^{2} t} \sinh (\pi \theta)},
$$


and depends on the inner product $t=\langle\xi, \eta\rangle$ of the arguments $\xi, \eta \in \mathbb{S}^{2}$. For $\gamma>0$, we define:

$$
U(\gamma):=\frac{1}{4 \pi} \int_{-\infty}^{+\infty} n(\gamma, \widehat{\mathbf{S}}(\theta)) d \theta
$$

This function was studied in detail in [7]; it is used in the existence proof for the Efimov effect. In particular, the function $U(\cdot)$ is continuous in $\gamma>0$, and the limit:

$$
\lim _{\mathbf{r} \rightarrow 0} \frac{1}{2} \mathbf{r}^{-1} n\left(\gamma, \mathbf{S}_{\mathbf{r}}\right)=U(\gamma)
$$

exists such that $U(1)>0$. lemma.

Theorem 3.3 can be derived by using a perturbation argument based on the following

Lemma 6.1. Let $A(z)=A_{0}(z)+A_{1}(z)$, where $A_{0}(z)\left(A_{1}(z)\right)$ is compact and continuous for $z<0$ (for $z \leq 0)$. Assume that the limit $\lim _{z \rightarrow-0} f(z) n\left(\gamma, A_{0}(z)\right)=l(\gamma)$ exists and $l(\cdot)$ is continuous in $(0 ;+\infty)$ for some function $f(\cdot)$, where $f(z) \rightarrow 0$ as $z \rightarrow-0$. Then, the same limit exists for $A(z)$ and $\lim _{z \rightarrow-0} f(z) n(\gamma, A(z))=l(\gamma)$.

For the proof of Lemma 6.1, see Lemma 4.9 in [7].

Remark 6.2. Since the function $U(\cdot)$ is continuous with respect to $\gamma$, it follows from Lemma 6.1 that any perturbation of $A_{0}(z)$ treated in Lemma 6.1 (which is compact and continuous up to $z=0$ ) does not contribute to the asymptotic relation (3.2).

Now, we are going to reduce the study of the asymptotics for the operator $\widehat{\mathbf{T}}(\mathbf{0} ; z)$ to that of the asymptotics $\mathbf{S}_{\mathbf{r}}$.

Let $\mathbf{T}(\delta ;|z|)$ be the $3 \times 3$ block operator matrix in $L_{2}^{(3)}\left(\mathbb{T}^{3}\right)$ whose entries $T_{\alpha \beta}(\delta ;|z|)$ are integral operators with the kernel $T_{\alpha \beta}(\delta ;|z| ; \cdot, \cdot)$ :

$$
\begin{aligned}
& T_{\alpha \alpha}(\delta ;|z| ; p, q)=0 ; \\
& T_{\alpha \beta}(\delta ;|z| ; p, q)= \\
& D_{\alpha \beta} \sum_{p^{\prime}, q^{\prime} \in \Lambda} \frac{\chi_{\delta}\left(p-p^{\prime}\right) \chi_{\delta}\left(q-q^{\prime}\right)\left(n_{\alpha}\left|p-p^{\prime}\right|^{2}+2|z| /\left(n^{2}\right)\right)^{-\frac{1}{4}}\left(n_{\beta}\left|q-q^{\prime}\right|^{2}+2|z| /\left(n^{2}\right)\right)^{-\frac{1}{4}}}{m_{\alpha \gamma}^{-1}\left|p-p^{\prime}\right|^{2}+2 m_{\gamma}^{-1}\left(p-p^{\prime}, q-q^{\prime}\right)+m_{\beta \gamma}^{-1}\left|q-q^{\prime}\right|^{2}+2|z| /\left(n^{2}\right)},
\end{aligned}
$$

where

$$
D_{\alpha \beta}=\frac{m_{\alpha \gamma}^{-3 / 4} m_{\beta \gamma}^{-3 / 4}}{2 n^{3} \pi^{2}}, \quad \alpha, \beta, \gamma=1,2,3, \quad \alpha \neq \beta \neq \gamma,
$$

and $\chi_{\delta}(\cdot)$ is the characteristic function of the domain $U_{\delta}(\mathbf{0})$.

Lemma 6.3. Let $\mu_{\alpha}=\mu_{\alpha}^{0}, \mu_{\beta}=\mu_{\beta}^{0}, \mu_{\gamma} \leq \mu_{\gamma}^{0}$. For any $z \leq 0$ and sufficiently small $\delta>0$, the difference $\widehat{\mathbf{T}}(\mathbf{0} ; z)-\mathbf{T}(\delta ;|z|)$ belongs to the Hilbert-Schmidt class, and is continuous with respect to $z \leq 0$.

Proof. We prove the lemma in the case $\mu_{\alpha}=\mu_{\alpha}^{0}, \alpha=1,2,3$. The case $\mu_{\alpha}=\mu_{\alpha}^{0}, \mu_{\beta}=\mu_{\beta}^{0}$, $\mu_{\gamma}<\mu_{\gamma}^{0}$ can be proven similarly.

By the definition of $\varepsilon_{\alpha}(\cdot)$, we have:

$$
\varepsilon_{\alpha}(p)=\frac{n^{2}}{2 m_{\alpha}}\left|p-p^{\prime}\right|^{2}+O\left(\left|p-p^{\prime}\right|^{4}\right),
$$


as $\left|p-p_{i}\right| \rightarrow 0$ for $p^{\prime} \in \Lambda$, which implies the expansion:

$$
E_{K-p}^{(\alpha)}(q)+\varepsilon_{\alpha}(p)=n^{2}\left[\frac{\left|p-p^{\prime}\right|^{2}}{2 m_{\alpha \gamma}}+\frac{\left(p-p^{\prime}, q-q^{\prime}\right)}{m_{\gamma}}+\frac{\left|q-q^{\prime}\right|^{2}}{2 m_{\beta \gamma}}\right]+O\left(\left|p-p^{\prime}\right|^{4}\right)+O\left(\left|q-q^{\prime}\right|^{4}\right),
$$

as $\left|p-p^{\prime}\right|,\left|q-q^{\prime}\right| \rightarrow 0$ for $K, p^{\prime}, q^{\prime} \in \Lambda$. Then, there exist $C_{1}, C_{2}>0$ and $\delta>0$ such that:

$$
\begin{aligned}
& C_{1}\left(\left|p-p^{\prime}\right|^{2}+\left|q-q^{\prime}\right|^{2}\right) \leq E_{K-p}^{(\alpha)}(q)+\varepsilon_{\alpha}(p) \leq C_{2}\left(\left|p-p^{\prime}\right|^{2}+\left|q-q^{\prime}\right|^{2}\right), \\
& (p, q) \in U_{\delta}\left(p^{\prime}\right) \times U_{\delta}\left(q^{\prime}\right) \quad \text { for } \quad K, p^{\prime}, q^{\prime} \in \Lambda ; \\
& E_{K-p}^{(\alpha)}(q)+\varepsilon_{\alpha}(p) \geq C_{1}, \quad(p, q) \in \mathbb{T}_{\delta}^{2}, \quad K \in \mathbb{T}^{3} .
\end{aligned}
$$

Applying last estimates and Corollary 4.6, we obtain that there exists $C_{1}>0$ such that the kernel of the operator $\widehat{T}_{\alpha \beta}(\mathbf{0} ; z)-T_{\alpha \beta}(\delta ;|z|)$ can be estimated by the squareintegrable function:

$$
C_{1} \sum_{p^{\prime}, q^{\prime} \in \Lambda}\left[\frac{1}{\left|p-p^{\prime}\right|^{2}+\left|q-q^{\prime}\right|^{2}}+\frac{\left|p-p^{\prime}\right|^{-1 / 2}}{\left|p-p^{\prime}\right|^{2}+\left|q-q^{\prime}\right|^{2}}+\frac{\left|q-q^{\prime}\right|^{-1 / 2}}{\left|p-p^{\prime}\right|^{2}+\left|q-q^{\prime}\right|^{2}}+1\right] .
$$

Hence, the operator $\widehat{T}_{\alpha \beta}(\mathbf{0} ; z)-T_{\alpha \beta}(\delta ;|z|)$ belongs to the Hilbert-Schmidt class for all $z \leq 0$. In combination with the continuity of the kernel of the operator with respect to $z<0$, this implies the continuity of $\widehat{T}_{\alpha \beta}(\mathbf{0} ; z)-T_{\alpha \beta}(\delta ;|z|)$ with respect to $z \leq 0$. The lemma is proved.

The following theorem is fundamental for the proof of the asymptotic relation (3.2).

Theorem 6.4. The following relation holds

$$
\lim _{|z| \rightarrow 0} \frac{n(\gamma, \mathbf{T}(\delta ;|z|))}{|\log | z||}=U(\gamma), \quad \gamma>0 .
$$

Proof. First we prove Theorem 6.4 under the condition that all two-particle operators have zero energy resonances, that is, in the case where $\mu_{\alpha}=\mu_{\alpha}^{0}, \alpha=1,2,3$. The case where only two operators $h_{\alpha}(\mathbf{0})$ and $h_{\beta}(\mathbf{0})$ have zero energy resonance can be proven similarly.

The subspace of vector functions $w=\left(w_{1}, w_{2}, w_{3}\right)$ with components having support in $\bigcup_{p^{\prime} \in \Lambda} U_{\delta}\left(p^{\prime}\right)$ is an invariant subspace for the operator $\mathbf{T}(\delta ;|z|)$.

Let $\mathbf{T}_{0}(\delta ;|z|)$ be the restriction of the operator $\mathbf{T}(\delta ;|z|)$ to the subspace $L_{2}^{(3)}\left(\bigcup_{p^{\prime} \in \Lambda} U_{\delta}\left(p^{\prime}\right)\right)$, that is, a $3 \times 3$ block operator matrix in $L_{2}^{(3)}\left(\bigcup_{p^{\prime} \in \Lambda} U_{\delta}\left(p^{\prime}\right)\right)$ whose entries $T_{\alpha \beta}^{(0)}(\delta ;|z|)$ are the integral operators with the kernel $T_{\alpha \beta}^{(0)}(\delta ;|z| ; \cdot, \cdot)$, where $T_{\alpha \alpha}^{(0)}(\delta ;|z| ; p, q)=0$ and the function $T_{\alpha \beta}^{(0)}(\delta ;|z| ; \cdot, \cdot)$ is defined on $\bigcup_{p^{\prime} \in \Lambda} U_{\delta}\left(p^{\prime}\right) \times \bigcup_{q^{\prime} \in \Lambda} U_{\delta}\left(q^{\prime}\right)$ as:

$$
\begin{aligned}
& T_{\alpha \beta}^{(0)}(\delta ;|z| ; p, q)=D_{\alpha \beta} \frac{\left(n_{\alpha}\left|p-p^{\prime}\right|^{2}+2|z| /\left(n^{2}\right)\right)^{-\frac{1}{4}}\left(n_{\beta}\left|q-q^{\prime}\right|^{2}+2|z| /\left(n^{2}\right)\right)^{-\frac{1}{4}}}{m_{\alpha \gamma}^{-1}\left|p-p^{\prime}\right|^{2}+2 m_{\gamma}^{-1}\left(p-p^{\prime}, q-q^{\prime}\right)+m_{\beta \gamma}^{-1}\left|q-q^{\prime}\right|^{2}+2|z| /\left(n^{2}\right)}, \\
& (p, q) \in U_{\delta}\left(p^{\prime}\right) \times U_{\delta}\left(q^{\prime}\right) \text { for } p^{\prime}, q^{\prime} \in \Lambda .
\end{aligned}
$$

In the remainder of the proof, for convenience, we numerate the points of $\Lambda$ as $p_{1}, \ldots, p_{n^{3}}$ and set $\overline{1, n}=1, \ldots, n$. 
Since $L_{2}\left(\bigcup_{i=1}^{n^{3}} U_{\delta}\left(p_{i}\right)\right) \cong \bigoplus_{i=1}^{n^{3}} L_{2}\left(U_{\delta}\left(p_{i}\right)\right)$, we can express the integral operator $T_{\alpha \beta}^{(0)}(\delta ;|z|)$ as the following $n^{3} \times n^{3}$ block operator matrix $\mathbf{T}_{\alpha \beta}^{(0)}(\delta ;|z|)$ acting on $\bigoplus_{i=1}^{n^{3}} L_{2}\left(U_{\delta}\left(p_{i}\right)\right)$ as:

$$
\mathbf{T}_{\alpha \beta}^{(0)}(\delta ;|z|)=\left(\begin{array}{ccc}
T_{\alpha \beta}^{(1,1)}(\delta ;|z|) & \ldots & T_{\alpha \beta}^{\left(1, n^{3}\right)}(\delta ;|z|) \\
\vdots & \ddots & \vdots \\
T_{\alpha \beta}^{\left(n^{3}, 1\right)}(\delta ;|z|) & \ldots & T_{\alpha \beta}^{\left(n^{3}, n^{3}\right)}(\delta ;|z|)
\end{array}\right)
$$

where $T_{\alpha \beta}^{(i, j)}(\delta ;|z|): L_{2}\left(U_{\delta}\left(p_{j}\right)\right) \rightarrow L_{2}\left(U_{\delta}\left(p_{i}\right)\right)$ is an integral operator with the kernel $T_{\alpha \beta}^{(0)}(\delta ;|z| ; p, q),(p, q) \in U_{\delta}\left(p_{i}\right) \times U_{\delta}\left(p_{j}\right)$ for $i, j=\overline{1, n^{3}}$.

It is easy to show that $\mathbf{T}_{0}(\delta ;|z|)$ is unitarily equivalent to the $3 \times 3$ block operator matrix $\mathbf{T}(r), r=|z|^{-\frac{1}{2}}$, acting on $L_{2}^{\left(n^{3}\right)}\left(U_{r}(\mathbf{0})\right) \oplus L_{2}^{\left(n^{3}\right)}\left(U_{r}(\mathbf{0})\right) \oplus L_{2}^{\left(n^{3}\right)}\left(U_{r}(\mathbf{0})\right)$ with the entries $\mathbf{T}_{\alpha \beta}(r): L_{2}^{\left(n^{3}\right)}\left(U_{r}(\mathbf{0})\right) \rightarrow L_{2}^{\left(n^{3}\right)}\left(U_{r}(\mathbf{0})\right):$

$$
\mathbf{T}_{\alpha \alpha}(r)=0 ; \quad \mathbf{T}_{\alpha \beta}(r)=\left(\begin{array}{ccc}
T_{\alpha \beta}(r) & \ldots & T_{\alpha \beta}(r) \\
\vdots & \ddots & \vdots \\
T_{\alpha \beta}(r) & \ldots & T_{\alpha \beta}(r)
\end{array}\right),
$$

where $T_{\alpha \beta}(r)$ is the integral operator on $L_{2}\left(U_{r}(\mathbf{0})\right)$ with the kernel:

$$
D_{\alpha \beta} \frac{\left(n_{\alpha}|p|^{2}+2 /\left(n^{2}\right)\right)^{-\frac{1}{4}}\left(n_{\beta}|q|^{2}+2 /\left(n^{2}\right)\right)^{-\frac{1}{4}}}{m_{\alpha \gamma}^{-1}|p|^{2}+2 m_{\gamma}^{-1}(p, q)+m_{\beta \gamma}^{-1}|q|^{2}+2 /\left(n^{2}\right)} .
$$

The equivalence is realized by the unitary dilation $\left(3 n^{3} \times 3 n^{3}\right.$ diagonal matrix):

$$
\begin{aligned}
& \mathbf{B}_{r}: \bigoplus_{i=1}^{3 n^{3}} L_{2}\left(U_{\delta}\left(p_{i}\right)\right) \rightarrow L_{2}^{\left(3 n^{3}\right)}\left(U_{r}(\mathbf{0})\right) \\
& \mathbf{B}_{r}=\operatorname{diag}\left\{B_{r}^{(1)}, \ldots, B_{r}^{\left(n^{3}\right)}, B_{r}^{(1)}, \ldots, B_{r}^{\left(n^{3}\right)}, B_{r}^{(1)}, \ldots, B_{r}^{\left(n^{3}\right)}\right\} .
\end{aligned}
$$

Here, the operator $B_{r}^{(i)}: L_{2}\left(U_{\delta}\left(p_{i}\right)\right) \rightarrow L_{2}\left(U_{r}(\mathbf{0})\right), i=\overline{1, n^{3}}$ acts as:

$$
\left(B_{r}^{(i)} f\right)(p)=\left(\frac{r}{\delta}\right)^{-\frac{3}{2}} f\left(\frac{\delta}{r} p+p_{i}\right) .
$$

Let us introduce the $3 n^{3} \times 1$ and $1 \times 3 n^{3}$ block operator matrices:

$$
\mathbf{E}: L_{2}^{\left(3 n^{3}\right)}\left(U_{r}(\mathbf{0})\right) \rightarrow L_{2}^{(3)}\left(U_{r}(\mathbf{0})\right), \quad \mathbf{A}_{r}: L_{2}^{(3)}\left(U_{r}(\mathbf{0})\right) \rightarrow L_{2}^{\left(3 n^{3}\right)}\left(U_{r}(\mathbf{0})\right)
$$

of the form

$$
\mathbf{A}_{r}=\left(\begin{array}{ccc}
0 & \mathbf{A}_{12}(r) & \mathbf{A}_{13}(r) \\
\mathbf{A}_{21}(r) & 0 & \mathbf{A}_{23}(r) \\
\mathbf{A}_{31}(r) & \mathbf{A}_{32}(r) & 0
\end{array}\right), \quad \mathbf{E}=\operatorname{diag}\{\mathbf{I}, \mathbf{I}, \mathbf{I}\},
$$

where $\mathbf{A}_{\alpha \beta}(r)$ and $\mathbf{I}$ are the $n^{3} \times 1$ and $1 \times n^{3}$ matrices of the form:

$$
\mathbf{A}_{\alpha \beta}(r)=\left(\begin{array}{c}
T_{\alpha \beta}^{(1)}(r) \\
\vdots \\
T_{\alpha \beta}^{(1)}(r)
\end{array}\right), \quad \mathbf{I}=(I \ldots I)
$$

respectively, here $I$ is the identity operator on $L_{2}\left(U_{r}(\mathbf{0})\right)$. 
It is well known that if $B_{1}, B_{2}$ are bounded operators and $\gamma \neq 0$ is an eigenvalue of $B_{1} B_{2}$, then $\gamma$ is an eigenvalue for $B_{2} B_{1}$ as well of the same algebraic and geometric multiplicities (see e.g. [20]). Therefore, $n\left(\gamma, \mathbf{A}_{r} \mathbf{E}\right)=n\left(\gamma, \mathbf{E} \mathbf{A}_{r}\right), \gamma>0$. Note that $\mathbf{T}_{\alpha \beta}(r)=$ $\mathbf{A}_{\alpha \beta}(r) \mathbf{I}$ and: $n^{3} T_{\alpha \beta}(r)=\mathbf{I} \mathbf{A}_{\alpha \beta}(r)$. Hence, direct calculation shows that $\mathbf{T}(r)=\mathbf{A}_{r} \mathbf{E}$ and

$$
\mathbf{E A}_{r}: L_{2}^{(3)}\left(U_{r}(\mathbf{0})\right) \rightarrow L_{2}^{(3)}\left(U_{r}(\mathbf{0})\right), \quad \mathbf{E A}_{r}=n^{3}\left(\begin{array}{ccc}
0 & T_{12}^{(1)}(r) & T_{13}^{(1)}(r) \\
T_{21}^{(1)}(r) & 0 & T_{13}^{(1)}(r) \\
T_{31}^{(1)}(r) & T_{32}^{(1)}(r) & 0
\end{array}\right) .
$$

So, $n\left(\gamma, \mathbf{T}_{1}(r)\right)=n\left(\gamma, \mathbf{E} \mathbf{A}_{r}\right), \gamma>0$.

Furthermore, we can replace:

$$
\left(n_{\alpha}|p|^{2}+\frac{2}{n^{2}}\right)^{\frac{1}{4}},\left(n_{\beta}|q|^{2}+\frac{2}{n^{2}}\right)^{\frac{1}{4}} \text { and } \frac{|p|^{2}}{m_{\alpha \gamma}}+\frac{2(p, q)}{m_{\gamma}}+\frac{|q|^{2}}{m_{\beta \gamma}}+\frac{2}{n^{2}}
$$

by the expressions:

$$
\left(n_{\alpha}|p|^{2}\right)^{\frac{1}{4}}\left(1-\chi_{1}(p)\right)^{-1},\left(n_{\beta}|q|^{2}\right)^{\frac{1}{4}}\left(1-\chi_{1}(q)\right)^{-1} \text { and } \frac{|p|^{2}}{m_{\alpha \gamma}}+\frac{2(p, q)}{m_{\gamma}}+\frac{|q|^{2}}{m_{\beta \gamma}},
$$

respectively, because the corresponding difference is a Hilbert-Schmidt operator and continuous up to $z=0$. In this case, we obtain the block operator matrix $\mathbf{S}(r)$ on $L_{2}^{(3)}\left(U_{r}(\mathbf{0}) \backslash\right.$ $\left.U_{1}(\mathbf{0})\right)$ whose entries $S_{\alpha \beta}(r)$ are the integral operators with the kernel $S_{\alpha \beta}(r ; \cdot, \cdot)$ :

$$
S_{\alpha \alpha}(r ; p, q)=0 ; \quad S_{\alpha \beta}(r ; p, q)=\frac{n^{3} D_{\alpha \beta}}{\left(n_{1} n_{2}\right)^{1 / 4}} \frac{|p|^{-1 / 2}|q|^{-1 / 2}}{m_{\alpha \gamma}^{-1}|p|^{2}+2 m_{\gamma}^{-1}(p, q)+m_{\beta \gamma}|q|^{2}} .
$$

Using the dilation:

$$
\mathbf{M}=\operatorname{diag}\{M, M, M\}: L_{2}^{(3)}\left(U_{r}(\mathbf{0}) \backslash U_{1}(\mathbf{0})\right) \rightarrow L_{2}\left((0, \mathbf{r}), \sigma^{(3)}\right), \quad(M f)(x, w)=e^{3 x / 2} f\left(e^{x} w\right),
$$

where $\mathbf{r}=\frac{1}{2}|\log | z||, x \in(0, \mathbf{r}), w \in \mathbb{S}^{2}$, one can see that the operator $\mathbf{S}(r)$ is unitarily equivalent to the integral operator $\mathbf{S}_{\mathbf{r}}$.

Since the difference of the operators $\mathbf{S}_{\mathbf{r}}$ and $\mathbf{T}(\delta ;|z|)$ is compact (up to unitary equivalence) and $\mathbf{r}=1 / 2|\log | z||$, we obtain the equality:

$$
\lim _{|z| \rightarrow 0} \frac{n(\gamma, \mathbf{T}(\delta ;|z|))}{|\log | z||}=\lim _{\mathbf{r} \rightarrow 0} \frac{1}{2} \mathbf{r}^{-1} n\left(\gamma, \mathbf{S}_{\mathbf{r}}\right), \quad \gamma>0 .
$$

Now Lemma 6.1 and the equality (6.1) completes the proof of Theorem 6.4.

Proof of Theorem 3.3. Let $\mu_{\alpha}=\mu_{\alpha}^{0}, \alpha=1,2,3$. Using Lemmas 6.1, 6.3 and Theorem 6.4 we have:

$$
\lim _{|z| \rightarrow 0} \frac{n(1, \widehat{\mathbf{T}}(z))}{|\log | z||}=U(1)
$$

Taking into account the last equality and Lemma 5.1, and setting $\mathcal{U}_{0}=U(1)$, we complete the proof of Theorem 3.3.

\section{Acknowledgements}

The authors would like to thank Prof. A. Teta for helpful discussions about the results of the paper. This work was supported by the TOSCA Erasmus Mundus grant. T.H. Rasulov wishes to thank the University of L'Aquila for the invitation and hospitality. 


\section{References}

[1] V. Efimov. Energy levels arising from resonant two-body forces in a three-body system. Phys. Lett. B, 33 (8), P. 563-564 (1970).

[2] S. Albeverio, R. Höegh-Krohn, T.T. Wu. A class of exactly solvable three-body quantum mechanical problems and the universal low energy behavior. Phys. Lett. A, 83 (3), P. 105-109 (1981).

[3] R.D. Amado, J.V. Noble. On Efimov's effect: a new pathology of three-particle systems. Phys. Lett. B, 35, P. 25-27 (1971); II. Phys. Lett. D, 5 (3), P. 1992-2002 (1972).

[4] D.R. Yafaev. On the theory of the discrete spectrum of the three-particle Schrödinger operator. Math. USSR-Sb., 23, P. 535-559 (1974).

[5] G.F. Dell'Antonio, R. Figari, A. Teta. Hamiltonians for systems of $N$ particles interacting through point interactions. Ann. Inst. Henri Poincaré, Phys. Theor., 60 (3), P. 253-290 (1994).

[6] Yu.N. Ovchinnikov, I.M. Sigal. Number of bound states of three-body systems and Efimov's effect. Ann. Phys., 123 (2), P. 274-295 (1979).

[7] A.V. Sobolev. The Efimov effect. Discrete spectrum asymptotics. Commun. Math. Phys., 156 (1), P. $101-126$ (1993).

[8] H. Tamura. The Efimov effect of three-body Schrödinger operators. J. Func. Anal., 95 (2), P. 433-459 (1991).

[9] H. Tamura. The Efimov effect of three-body Schrödinger operators: asymptotics for the number of negative eigenvalues. Nagoya Math. J., 130, P. 55-83 (1993).

[10] D. Mattis. The few-body problem on a lattice. Rev. Modern Phys., 58 (2), P. 361-379 (1986).

[11] A.I. Mogilner. Hamiltonians in solid state physics as multiparticle discrete Schrödinger operators: problems and results. Advances in Sov. Math., 5, P. 139-194 (1991).

[12] M. Reed, B. Simon. Methods of Modern Mathematical Physics. III: Scattering theory. Academic Press, New York, 1979.

[13] V.A. Malishev, R.A. Minlos. Linear infinite-particle operators. Translations of Mathematical Monographs. 143, AMS, Providence, RI, 1995.

[14] L.D. Faddeev. Mathematical aspects of the three-body problem in quantum mechanics. Israel Program for Scientific Translations, Jerusalem, 1965.

[15] L.D. Faddeev, S.P. Merkuriev. Quantum scattering theory for several particle systems. Kluwer Academic Publishers, 1993.

[16] Zh.I. Abdullaev, S.N. Lakaev. Asymptotics of the discrete spectrum of the three-particle Schrödinger difference operator on a lattice. Theor. Math. Phys., 136 (2), P. 1096-1109 (2003).

[17] S. Albeverio, S.N. Lakaev, Z.I. Muminov. Schrödinger operators on lattices. The Efimov effect and discrete spectrum asymptotics. Ann. Henri Poincaré, 5, P. 743-772 (2004).

[18] S.N. Lakaev, M.É. Muminov. Essential and discrete spectra of the three-particle Schrödinger operator on a lattices. Theor. Math. Phys., 135 (3), P. 849-871 (2003).

[19] Zh.I. Abdullaev. Finiteness of the discrete spectrum for non-trivial of the full quasi-momentum in the system of three bosons on a lattice. Russian Math. Surveys, 62 (1), P. 175-201 (2007).

[20] P.R. Halmos. A Hilbert space problem book. Springer-Verlag New York Inc., second edition, 1982.

[21] M.I. Muminov. Positivity of the two-particle Hamiltonian on a lattice. Theoret. Math. Phys., 153 (3), P. $1671-1676$ (2007). 